\title{
Collision probabilities of migrating small bodies and dust particles with planets
}

\author{
Sergei I. Ipatov ${ }^{1,2}$ \\ ${ }^{1}$ Catholic University of America \\ Washington DC, USA \\ email: siipatov@hotmail.com \\ ${ }^{2}$ Space Research Institute, Moscow, Russia
}

\begin{abstract}
Probabilities of collisions of migrating small bodies and dust particles produced by these bodies with planets were studied. Various Jupiter-family comets, Halley-type comets, longperiod comets, trans-Neptunian objects, and asteroids were considered. The total probability of collisions of any considered body or particle with all planets did not exceed 0.2 . The amount of water delivered from outside of Jupiter's orbit to the Earth during the formation of the giant planets could exceed the amount of water in Earth's oceans. The ratio of the mass of water delivered to a planet by Jupiter-family comets or Halley-type comets to the mass of the planet can be greater for Mars, Venus, and Mercury, than that for Earth.
\end{abstract}

Keywords. Minor planets, asteroids; comets: general; solar system: general

\section{Model of migration of small bodies and dust particles}

In the present paper we discuss the probabilities of collisions of migrating small bodies and dust particles produced by these bodies with planets. Ipatov \& Mather (2003, 2004ab, 2006, 2007), Ipatov et al. (2004), and several other authors cited in the above papers studied the probabilities with Earth, Venus, and Mars. In our above papers (which can be found on astro-ph and http://faculty.cua.edu/ipatov/list-publications.htm), the probabilities of the collisions were calculated based on the time variations of orbits of bodies and particles during their dynamical lifetimes (until all bodies or particles reached 2000 AU from the Sun or collided with the Sun). Using the same time variations, below we consider also the probabilities of collisions with the giant planets and Mercury.

The orbital evolution of $>30,000$ bodies with initial orbits close to those of Jupiterfamily comets (JFCs), Halley-type comets, long-period comets, trans-Neptunian objects, and asteroids in the resonances $3 / 1$ and $5 / 2$ with Jupiter, and also of $>20,000$ dust particles produced by these small bodies was integrated during their dynamical lifetimes. We considered the gravitational influence of planets, but omitted the influence of Mercury (exclusive for Comet 2P/Encke). In about a half of calculations of migration of bodies, we used the method by Bulirsh-Stoer (1966) (BULSTO code), and in other runs we used a symplectic method (RMVS3 code). The integration package of Levison \& Duncan (1994) was used. For dust particles, only the BULSTO code was used, and the gravitational influence of all planets, the Poynting-Robertson drag, radiation pressure, and solar wind drag were taken into account. The ratio $\beta$ between the radiation pressure force and the gravitational force varied from $\leqslant 0.0004$ to 0.4 . For silicates, such values of $\beta$ correspond to particle diameters $d$ between $\geqslant 1000$ and 1 microns; $d$ is proportional to $1 / \beta$.

In our calculations, planets were considered as material points, so literal collisions did not occur. However, using the algorithm suggested by Ipatov (1988) with the correction that takes into account a different velocity at different parts of the orbit (Ipatov \& Mather 2003), and based on all orbital elements sampled with a 10-500 yr step, we calculated 
the mean probability $P$ of collisions of migrating objects with a planet. The step could be different for different typical dynamical lifetimes of particles. We define $P$ as $P_{\Sigma} / N$, where $P_{\Sigma}$ is the probability of a collision of all $N$ objects with a planet. The probabilities of collisions of bodies and particles at different $\beta$ with planets are presented in Fig. 1. These probabilities do not take into account the destruction of particles in collisions and sublimation, which can be more important for small particles. Our runs were made mainly for direct modelling of collisions with the Sun, but Ipatov \& Mather (2007) obtained that the mean probabilities of collisions of considered bodies with planets, lifetimes of the bodies that spent millions of years in Earth-crossing orbits, and other obtained results were practically the same if we consider that bodies disappear when perihelion distance becomes less than the radius of the Sun or even several such radii.

\section{Probabilities of collisions of migrating small bodies and dust particles with planets and the Sun}

The probability $P_{E}$ of a collision of a JFC with the Earth exceeded $4 \cdot 10^{-6}$ if initial orbits of bodies were close to those of several tens of JFCs, even excluding a few bodies for which the probability of a collision of one body with the Earth could be greater than the sum of probabilities for thousands of other bodies. The Bulirsh-Stoer method of integration and the symplectic method gave similar results. The ratios of probabilities of collisions of JFCs with Venus, Mars, and Mercury to the mass of a planet usually were not smaller than those for Earth. For most considered bodies, the probabilities $P_{M e}$ of collisions of most bodies with Mercury (exclusive for Comet 2P/Encke, for which $\left.P_{M e} \sim P_{E}\right)$ were smaller by an order of magnitude than those with Earth or Venus.

For dust particles produced by comets and asteroids, $P_{E}$ was found to have a maximum $(\sim 0.001-0.02)$ at $0.002 \leqslant \beta \leqslant 0.01$, i.e., at $d \sim 100 \mu \mathrm{m}$ (this value of $d$ is in accordance with observational data). These maximum values of $P_{E}$ were usually (exclusive for Comet $2 \mathrm{P} /$ Encke) greater at least by an order of magnitude than the values for parent comets. Probabilities of collisions of most considered particles with Venus did not differ much from those with Earth, and those with Mars were about an order of magnitude smaller. For particles produced by Halley-type comets, $P$ was greater for Mercury than for Mars.

Using $P_{E}=4 \cdot 10^{-6}$ and assuming that the total mass of planetesimals that ever crossed Jupiter's orbit was about $100 m_{E}$ (Ipatov 1987, 1993), where $m_{E}$ is the mass of the Earth, Ipatov \& Mather (2003, 2004a-b, 2007) concluded that the total mass of water delivered from the feeding zone of the giant planets to the Earth could be about the total mass of water in Earth's oceans. (Similar conclusion was made by Ipatov (2001) based on other calculations.) We supposed that the fraction of water in planetesimals equaled 0.5. The ratio of the mass of water delivered to a planet by Jupiter-family comets and Halley-type comets to the mass of the planet can be greater for Mars, Venus, and Mercury, than that for Earth. This larger mass fraction would result in relatively large ancient oceans on Mars and Venus. The larger value of $P$ for Earth we have calculated compared to those argued by Morbidelli et al. (2000) $\left(P_{E} \sim(1-3) \cdot 10^{-6}\right)$ and Levison et al. (2001) $\left(P_{E} \sim 4 \cdot 10^{-7}\right)$ is caused by the fact that Levison et al. (2001) did not take into account the gravitational influence of the terrestrial planets, and Morbidelli et al. (2000) considered low-eccentric initial orbits beyond Jupiter's orbit. Besides, we considered a larger number of bodies. The detailed discussion on delivery of water and the comparison of our results with the results by other authors were made by Ipatov \& Mather (2007). Marov \& Ipatov (2005) discussed the delivery of volatiles to the terrestrial planets.

At the present time, most authors (e.g., Lunine et al. 2003, Morbidelli et al. 2000, and Petit et al. 2001) consider that the outer asteroid belt was the main source of the delivery 

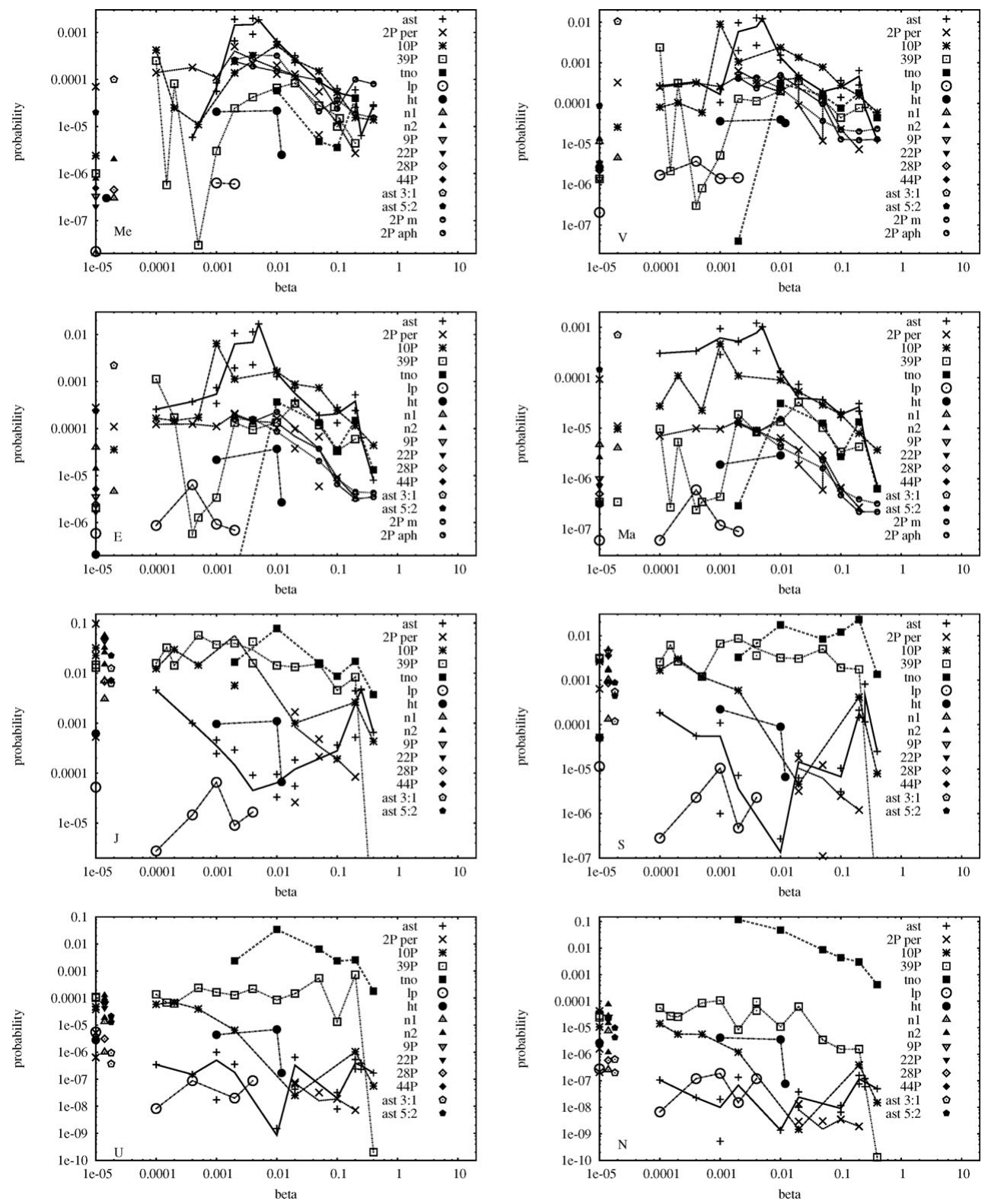

Figure 1. The probability of collisions of dust particles and bodies (during their dynamical lifetimes) with Mercury (subfigure Me), Venus (V), Earth (E), Mars (Ma), Jupiter (J), Saturn $(\mathrm{S})$, Uranus $(\mathrm{U})$, and Neptune $(\mathrm{N})$ versus $\beta$ (the ratio of the radiation to gravitational forces) for particles launched from asteroids (ast), trans-Neptunian objects (tno), Comet 2P/Encke at perihelion $(2 \mathrm{P}$ per $)$, Comet $2 \mathrm{P} /$ Encke at aphelion $(2 \mathrm{P}$ aph), Comet $2 \mathrm{P} /$ Encke in the middle between perihelion and aphelion $(2 \mathrm{P} \mathrm{m})$, Comet 10P/Tempel $2(10 \mathrm{P})$, Comet 39P/Oterma $(39 \mathrm{P})$, long-period comets $(l p)$ at eccentricity $e=0.995$ and perihelion distance $q=0.9 \mathrm{AU}$, and Halley-type comets $(h t)$ at $e=0.975$ and $q=0.5 \mathrm{AU}$ (for $l p$ and $h t$ runs, initial inclinations were from 0 to $180^{\circ}$, and particles were launched near perihelia). If there are two points for the same $\beta$, then a plot is drawn via their mean value. Probabilities presented at $\beta \sim 10^{-5}$ are for small bodies $(\beta=0)$. Probabilities presented only for bodies were calculated for initial orbits close to orbits of Comets 9P/Tempel 1 (9P), 22P/Kopff (22P), 28P/Neujmin (28P), 44P/Reinmuth 2 $(44 \mathrm{P})$, and test asteroids from resonances $3: 1$ and $5: 2$ with Jupiter at $e=0.15$ and $i=10^{\circ}$ ('ast $3: 1^{\prime}$ and 'ast $5: 2$ '). For series $n 1$ and $n 2$, initial orbits of bodies were close to 10-20 different Jupiter-family comets (Ipatov \& Mather 2004b). 
of water to the terrestrial planets. Drake \& Campins (2006) noted that the key argument against an asteroidal source of Earth's water is that the O's isotopic composition of Earth's primitive upper mantle matches that of anhydrous ordinary chondrites, not hydrous carbonaceous chondrites. To the discussion of the deuterium/hydrogen paradox of the Earth's oceans presented by Ipatov \& Mather (2007), we can add that Genda \& Ikoma (2008) showed that D/H in the Earth's ocean increased by a factor of 2-9.

Probabilities of collisions of JFCs with Saturn typically were smaller by an order of magnitude than those with Jupiter, and collision probabilities with Uranus and Neptune typically were smaller by three orders of magnitude than those with Jupiter. As only a small fraction of comets collided with all planets during dynamical lifetimes of comets, the orbital evolution of comets for the considered model of material points was close to that for the model when comets collided with a planet are removed from integrations.

Probabilities of collisions of considered particles and bodies with Jupiter during their dynamical lifetimes are smaller than 0.1 . They can reach $0.01-0.1$ for bodies and particles initially moved beyond Jupiter's orbit or in Encke-type orbits. For bodies and particles initially moved inside Jupiter's orbit, the probabilities are usually smaller than the above range and can be zero. Probabilities of collisions of migrating particles (exclusive for trans-Neptunian particles) with other giant planets were usually smaller than those with Jupiter. The total probability of collisions of any considered body or particle with all planets did not exceed 0.2 .

Collisions of planetesimals with a star can cause variations in observed brightness and spectrum of the star. In our calculations, the fraction $P_{S u n}$ of comets collided with the Sun during their dynamical lifetimes was about a few percent. For most JFCs, dynamical lifetimes are less than $10 \mathrm{Myr}$, and on average $P_{\text {Sun }} \sim 0.02$. For dust particles, $P_{S u n}$ depends on $\beta$ and can be considerably greater than for their parent bodies. For example, for Comet $10 \mathrm{P} /$ Tempel $2, P_{S u n} \approx 0.01$, and almost all particles produced by this comet collide with the Sun (Ipatov \& Mather 2006).

\section{References}

Drake, M. \& Campins, H. 2006, in: D. Lazzaro, S. Ferraz-Mello \& J. A. Fernandez (eds), Asteroids, Comets, 83 Meteors, IAU Symp. 229 (Cambridge: Cambridge Univ. Press), p. 381

Genda, H. \& Icoma, M. 2008, Icarus, 194, 42

Ipatov, S. I. 1987, Earth, Moon, ES Planets, 39, 101

Ipatov, S. I. 1988, Sov. Astron., 65, 1075

Ipatov, S. I. 1993, Solar System Res., 27, 65

Ipatov, S. I. 2001, Adv. Space Res., 28, 1107

Ipatov, S. I. \& Mather, J. C. 2003, Earth, Moon, \& Planets, 92, 89

Ipatov, S. I. \& Mather, J. C. 2004a, Ann. New York Acad. Sci., 1017, 46

Ipatov, S. I. \& Mather, J. C. 2004b, Adv. Space Res., 33, 1524

Ipatov, S. I. \& Mather, J. C. 2006, Adv. Space Res., 37, 126

Ipatov, S. I. \& Mather, J. C. 2007, in: IAUS 236 Near-Earth Objects, Our Celestial Neighbors: Opportunity and Risk, p. 55

Ipatov, S. I., Mather, J. C., \& Taylor, P. A. 2004, Ann. New York Acad. Sci., 1017, 66

Levison, H. F. \& Duncan, M. J. 1994, Icarus, 108, 18

Levison, H. F., et al. 2001, Icarus, 151, 286

Lunine, J. I., Chambers, J., Morbidelli, A., \& Leshin, L. A. 2003, Icarus, 165, 1

Marov, M. Ya. \& Ipatov, S. I. 2006, Solar Syst. Res., 39, 374

Morbidelli, A., Chambers, J., Lunine, J. I., Petit, J. M., Robert, F., Valsecchi, G. B., \& Cyr, K. E. 2000, Meteoritics \& Planet. Sci., 35, 1309

Petit, J.-M., Morbidelli, A., \& Chambers, J. 2001, Icarus, 153, 338 